\title{
An OA-based integrated information management system building for marine monitoring business processes
}

\author{
Duyi Jiang \\ Key Laboratory of Marine Spill Oil \\ Identification and Damage \\ Assessment Technology, SOA \\ North China Sea Environmental \\ Monitoring Center, SOA \\ Qingdao, Shandong, China \\ done2004@126.com
}

\author{
Ting Zhang \\ Department of Computer Science \\ and Technology \\ Ocean University of China \\ Qingdao, China \\ tingzhangouc@126.com
}

\author{
Liang Qu \\ Key Laboratory of Marine Spill Oil \\ Identification and Damage \\ Assessment Technology, SOA \\ North China Sea Environmental \\ Monitoring Center, SOA \\ Qingdao, Shandong, China \\ ql.ocean@yahoo.com.cn
}

\begin{abstract}
Due to the increasing frequency and intensity of marine monitoring tasks, the need for a comprehensive information management system for marine monitoring to organize and implement all types of marine monitoring tasks is growing. This paper put forward an integrated information management system for marine monitoring using office automation (OA) technology which is based on service-oriented architecture (SOA) workflow. The system realized the management of monitoring tasks in all aspects and the statistical analysis of data. And it informationized the set of processes required.
\end{abstract}

Keywords-OA; marine monitoring business processes; integrated information management system

\section{INTRODUCTION}

Currently, the status of marine environmental monitoring in marine work is increasingly prominent. The sustainable development advocated by marine career development requires objectively paying close attention to the quality status of the marine environment [1] [2]. Some unexpected events, such as a variety of oil spill Enteromorpha outbreak and catastrophic events like the nuclear radiation leak influencing the ocean in Fukushima also need marine environmental monitoring. Marine monitoring departments according to the higher requirements must for the first time reach the waters that the event requires monitoring and implement the monitoring tasks. And environmental quality assessment report submitted to the higher authorities must be generated timely.

Marine environmental monitoring activities mainly refer to the design management and implementation of marine monitoring tasks with the sea team for the management unit led by a professional tour guides and comprised of marine chemistry, marine hydrology, marine biology, marine remote sensing and other technical staff [3] [4]. For example: determining the implementation plan, issuing the mission statement, determining the leader and personnel of the sea team, the organization and management of the supplies requisitioning and equipment, laboratory analysis and data audit, monitoring data analysis, the preparation of the corresponding report and atlas. Each task in marine monitoring must be designed to perform to meet the uniform requirements, which are fixed property of the mission on the sea. Marine monitoring activities are usually performed after inclusion in the annual plan. Such as: the process designs of marine monitoring activities need to be represented by chart with words; it is allowed to implement the mission on the sea and other follow-up work only after getting the various requirements of the tasks and the approval of the higher level.

Due to the increasing number of monitoring tasks, multiple tasks are conducted simultaneously often. However, there is lack of convenient and effective communication between different teams that conduct monitoring tasks. Thus, it is difficult to carry out effective integrated management.

The system realized the management of monitoring tasks in all aspects from issuing the mission statement to conducting the plan with the approval and the statistical analysis of marine environmental monitoring data. And it informationized the set of processes required.

\section{SYSTEM ARCHITECTURE}

This paper put forward an integrated information management system for marine monitoring using office automation (OA) [5] [6] technology which is based on service-oriented architecture (SOA) [7] [8] workflow. The system develops from an OA system that has B/S three layer architecture based on workflow [9] [10].

Specifically, the first step is to establish monitoring data and information management database and operating tables for monitoring tasks on the database server, and then to create a workflow engine and marine data management sub modules on the application server side with scripting language, the final is to present in the browser in the form of HTML pages. In this study, the system architecture is as follows: 

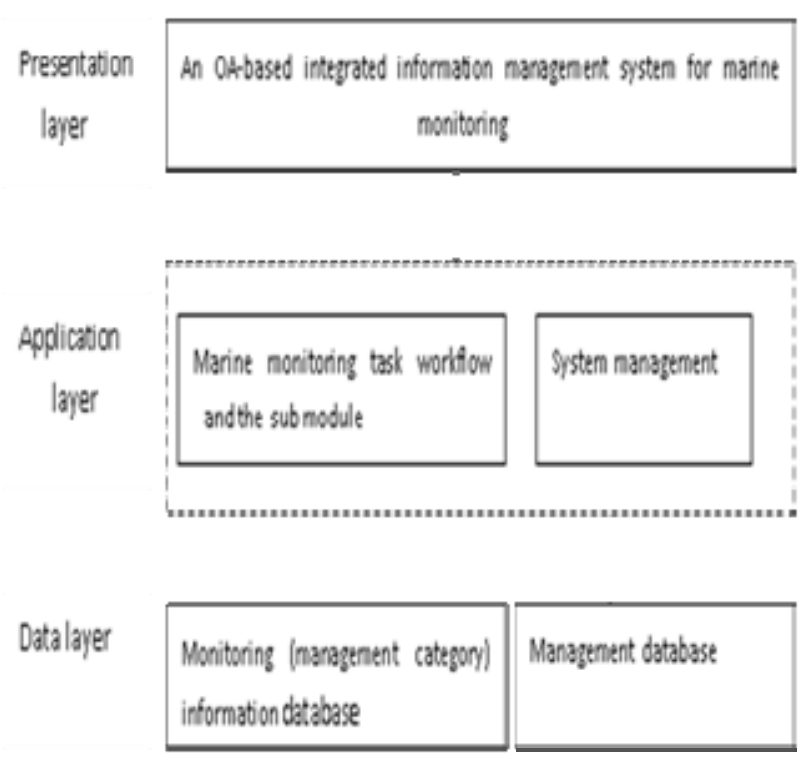

Figure 1. The overall structure drawing of operation management system.

\section{MAIN FUNCTIONS OF THE SYSTEM}

The system presented in the paper main include the following modules: system management, monitoring data query, command task management, approval process, scientific research management, document online editing, instant messaging, announcement and so on.

\section{A. System Management}

This is the key module of the entire system. Thus, organizational structure setting, workflow setting, administrative office setting and knowledge management setting are all completed in this module. Workflow refers to the abstraction, generalization and description of work process, as well as business rules among operation steps. And workflow modeling is about establishing an appropriate model in the computer to represent and implement the logic and rules which organize assignments together across the whole process. Marine environmental monitoring activities involve administrative class, the class of tasks, personnel, financial, research and other types of processes. And the function of workflow setting implements the design of the processes described above.

\section{B. Monitoring Data Query}

The module in this part has realized the query function of the North Sea environmental monitoring data so as to provide quick access to the environmental monitoring data for the relevant personnel. Authorized users can obtain screening results by setting query criteria and selecting the display items required. Function interface is shown in fig. 2.

Then query criteria include task type, monitoring task, monitoring content, monitoring element, the date of task, latitude and longitude, etc. In order to offer more humane service for users, the latitude and longitude can be determined by map. In addition, the items displayed in the query results can be selected by users, to further increase the usefulness of the system.

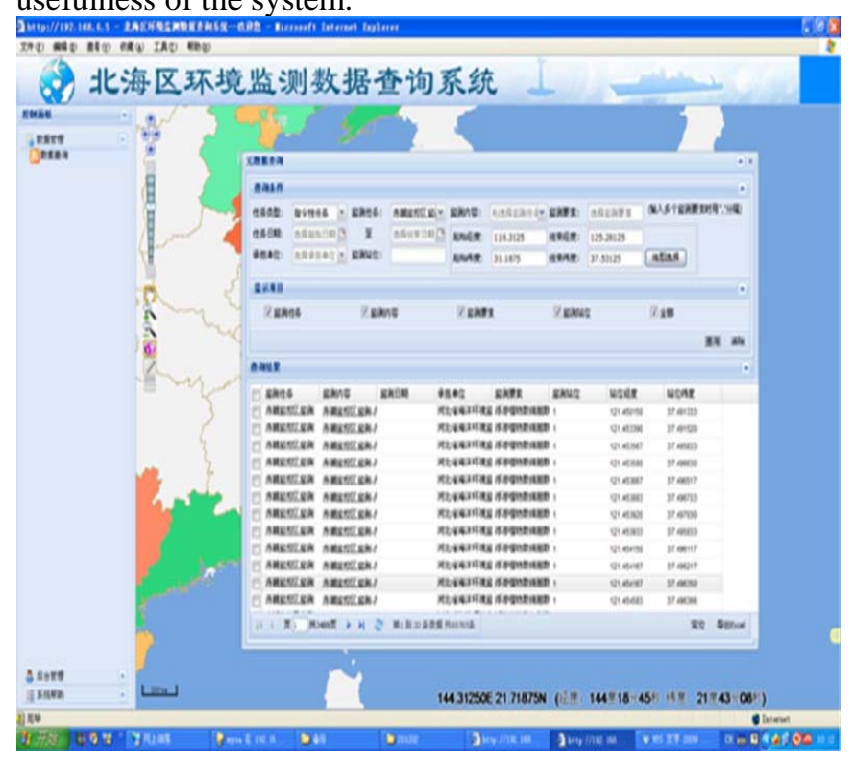

Figure 2. The query module of environmental monitoring data.

\section{Command Task Management}

Command task management subsystem includes three main features: new mandatory task, mandatory task query, management personnel designation. New mandatory task: the user through the initialization of the mandatory task information, create a new command task. The basic information of task are whether task is shared or not and shared scope (the scope of the project), project categories, project codes, project area, project name, etc. Mandatory task query: users can get shortcuts and obtain results needed with setting the query criteria, such as setting the project's scope like managers to view or shared projects. Authorized personnel also can edit, delete, and do other operations on query results.

\section{Approval Process}

The approval process module implements the function of new flow chart, workflow query, flow monitoring, and data report. Flow monitoring is a very important link, through which user can view the current step and organizer of monitoring process selected, as well as transfer and entrust the selected flow. The following fig. 3shows the monitoring of all flows receiving files. The design drawing of receiving files flow is shown in fig. 4 and the editing of flows is completed in the system management module. 


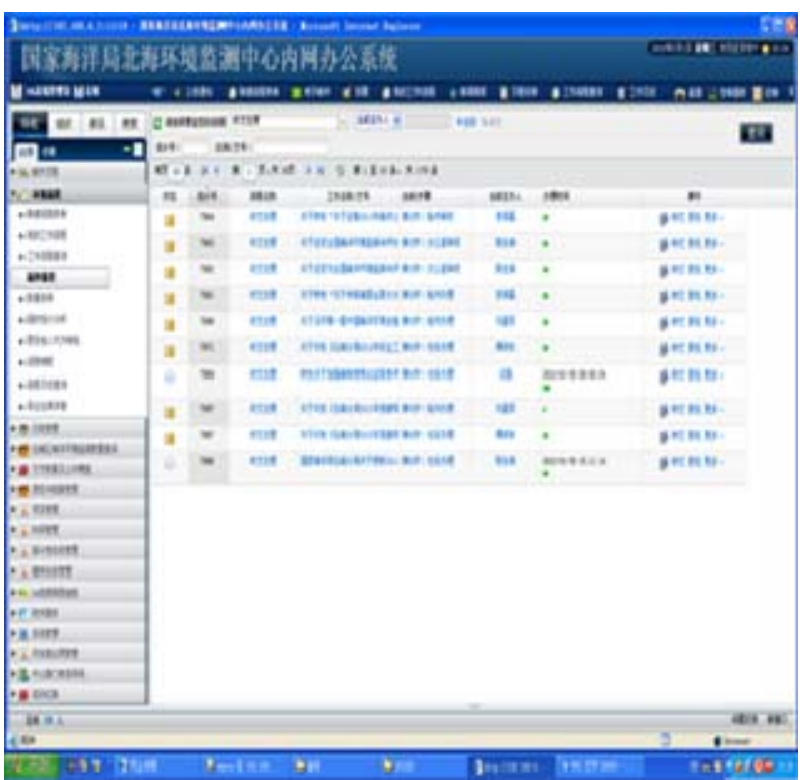

Figure 3. Approval process — the monitoring of all flows receiving files.

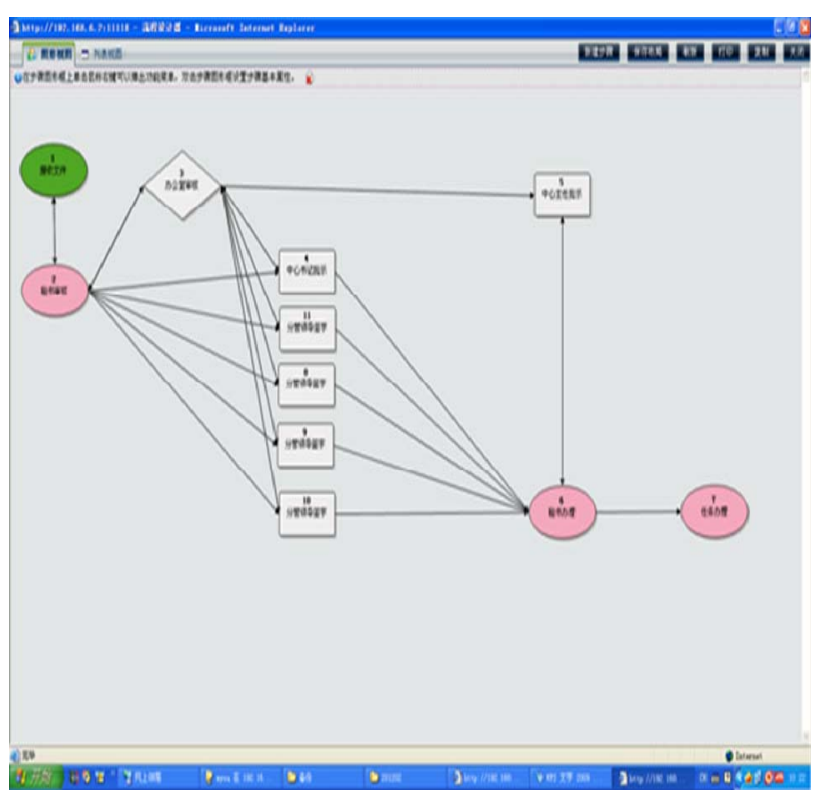

Figure 4. The design drawing of receiving files flow.

\section{E. Scientific Research Management}

This module has implemented the management of research projects and research achievements, the supervision and examination of projects. Users can add a research project (research achievement, supervision and examination information), manage research projects (research achievements, supervision and examination information), also manage and designate staff. The layout of each function is shown in fig. 5 .

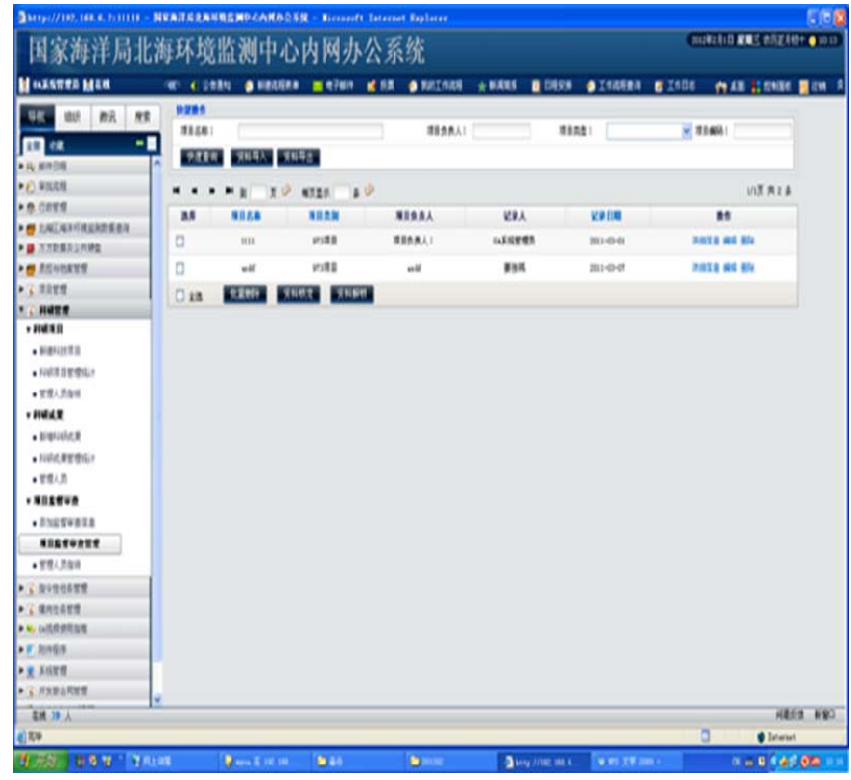

Figure 5. Scientific research management.

\section{F. Document Online Editing, Instant Messaging, Announcement}

Since these functions are common features of many OA systems, introduction in detail we owe you. Edit the document online: online editing supports for office documents (word, excel, power point documents) with not having to download and automatically uploading; it also supports for mark keeping, handwritten signature, electronic seal with the electronic seal verification function to ensure document security. Instant messaging: instant messaging tool similar with QQ is provided, which realizes the realtime communication and file transfer among users. Announcement: various notifications change from paper documents into pdf format and are sent as attachment under the releasing scope is optional.

\section{CONCLUSION}

The system in this paper implements effective management of marine monitoring information and affords assistant decision support functions. It provides technical support for marine environmental monitoring data query, analysis and various types of schemes generation and evaluation, provides a strong reference to the relevant administrative departments with decision-making in the management of the marine environmental monitoring activities, also effectively improves the work efficiency and public information services of the sea-related administrative departments.

\section{ACKNOWLEDGMENT}

This research was financially supported by the National Natural Science Foundation of China (NO. 40976002) and the Marine Science and Technology Project of the North Sea Branch, State Oceanic Administration: An OA-based 
integrated management information system for marine monitoring(NO. 2012B04).

\section{REFERENCES}

[1] J. Davies, J. Baxter, M. Bradley, D. Connor, J. Khan, E. Murray, W. Sanderson, C. Turnbull, and M. Vincent, Marine monitoring handbook. Joint Nature Conservation Committee, Peterborough, United Kingdom, March 2001.

[2] J.S. Gray, D. Calamari, R. Duce, J.E. Portmann, P.G. Wells, Scientifically based strategies for marine environmental protection and management, Marine Pollution Bulletin MPNBAZ, Vol. 22, No. 9, pp. 432-440, September 1991.

[3] D.E Wells, W.P Cofino, P Quevauviller, B Griepink, Quality assurance of information in marine monitoring: A holistic approach, Marine Pollution BulletinVol. 26, Issue 7, pp. 368-375, July 1993.

[4] P. Brasseur, P. Bahurel, L. Bertino, F. Birol, J.-M. Brankart, N. Ferry, S. Losa, E. Remy, J. Schröter, S. Skachko, C.-E. Testut, B. Tranchant, P. J. Van Leeuwen, J. Verron, Data assimilation for marine monitoring and prediction: The MERCATOR operational assimilation systems and the MERSEA developments, Quarterly Journal of the Royal Meteorological Society, Vol. 131, Issue 613, pp. 3561-3582, October 2005 Part C.

[5] Changkun QIN, Guanghua ZHU, Design and Implementation of Enterprise Office Automatic System, Computer and Modernization, 2003.

[6] Pei CUI, Yu ZHANG, WebGIS-Based Office Automation System, CD Technology, 2006.

[7] Thomas Erl, SOA: Principles of Service Design, Upper Saddle Creek, NJ, Prentice Hall, 2007.

[8] N. Josuttis, SOA in Practice: The Art of Distributed System Design, O’Reilly Media, Sebastopol, 2007.

[9] Frank Leymann, Dieter Roller, Production workflow: concepts and techniques, Prentice Hall PTR, Upper Saddle River, NJ, 1999.

[10] W.M.P. van der Aalst, S. Jablonski, Dealing with workflow change: Identification of issues and solutions, International Journal of Computer Systems, Science, and Engineering, 15 (5), pp. 267-276, 2000. 